\title{
COMMENTARY
}

\section{Clinical features of H1N1 2009 infection in critically ill immunocompromised patients}

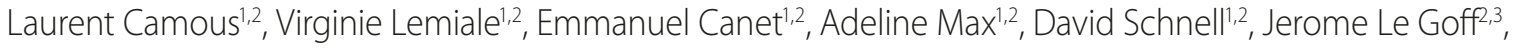 \\ Antoine Rabbatt, Benoit Schlemmer ${ }^{1,2}$ and Élie Azoulay ${ }^{* 1,2}$
}

\begin{abstract}
Seasonal influenza virus has been described as an emerging and severe pathogen in immunocompromised hosts. Since the beginning of the 2009 influenza A novel H1N1 pandemic, several series have described the clinical course of the disease in various populations. We report the clinical course of H1N1 2009 infection in 10 immunocompromised patients. Half of the patients received long-term steroid therapy. Disease was characterized by a clinical picture similar to that of non-immunocompromised patients but with prolonged course and higher mortality.
\end{abstract}

Infection is a major source of morbidity and the leading cause of death in immunocompromised patients [1]. The increased susceptibility to infection results from the intertwined effects of the immunocompromising condition, treatments, and co-morbidities [1]. Human infection with the novel H1N1 influenza virus was first recognized in early April 2009 and declared a worldwide pandemic by the World Health Organization in June 2009. Recent case series provide information on the clinical course, risk factors, and outcome of H1N1(v) infection [2-4]. Both New Zealand and Canada have experienced H1N1(v) outbreaks with severe illness requiring intensive care unit (ICU) admission, ventilatory support, and rescue therapies. However, no case series have specifically described the features of $\mathrm{H} 1 \mathrm{NI}(\mathrm{v})$ infection in immunocompromised patients. Here, we report the clinical and epidemiologic features in 10 critically ill immunocompromised patients with H1N1(v) infection.

*Correspondence: elie.azoulay@sls.aphp.fr

'Medical Intensive Care Unit, Saint-Louis University Hospital, APHP, 1 Avenue Claude Vellefaux, 75010 Paris, France

2Paris-7 Paris-Diderot University, UFR de Médecine, 1 Avenue Claude Vellefaux, 75010 Paris, France

Full list of author information is available at the end of the article
The case definition was ICU admission for acute respiratory failure and a positive specific polymerase chain reaction test for the pandemic influenza A (H1N1) 2009 virus. All patients meeting this case definition were included.

In late 2009, 15 patients with H1N1-related acute respiratory failure, including 10 immunocompromised patients, required ICU admission. As reported in Table 1, median time from respiratory symptom onset to ICU admission was 4 days (interquartile range [IQR] 3 to 5 days). Hypoxemia was mild at ICU admission but worsened over the next few days. The chest radiographs consistently showed extensive pulmonary infiltrates (median Murray score 3; IQR 2 to 4), and $80 \%$ of cases showed an alveolar pattern. All patients were treated with oseltamivir, which was prescribed 1 day (range 0 to 6 days) after ICU admission. Superinfection (mostly bacterial pneumonia) occurred in all patients in keeping with previous data on seasonal influenza [5]. The clinical course was characterized by prolonged oxygen dependency in the survivors (10 days; IQR 6 to 15 days). Death occurred in four patients and was usually secondary to severe hypoxemia.

H1N1(v) infection can result in a wide spectrum of clinical patterns, ranging from no symptoms to fulminant viral pneumonia. This new pandemic virus is characterized by a high prevalence of severe viral pneumonitis, which often requires mechanical ventilation [2]. Influenza viruses are known to cause severe infections in immunocompromised patients, of whom variable proportions were reported in epidemiologic descriptions [2-4]. Our case series is the first to describe the course of H1N1(v) infection in immunocompromised hosts.

Several points deserve to be highlighted. First, the risk factors for H1N1(v) described in the overall population [2-4] were not found in our cohort. In contrast, none of our patients had obesity (median body mass index 26.9; IQR 21 to 26) or chronic lung disease [3]. Of our 10 patients, 7 were on long-term steroid treatment, as described in the immunocompromised subgroup of the Canadian ICU patients [3]. Cellular immunodeficiency is the main risk factor for lower respiratory tract infection 
Table 1. Clinical characteristics and outcomes of H1N1(v) critically ill immunocompromised patients

\begin{tabular}{|c|c|c|c|c|c|c|c|c|c|}
\hline Patient & $\begin{array}{l}\text { Type of } \\
\text { immune } \\
\text { deficiency }\end{array}$ & nemotherapy & $\begin{array}{l}\text { Immuno- } \\
\text { suppressive } \\
\text { agents }\end{array}$ & $\begin{array}{l}\text { Lymphocyte } \\
\text { count }\end{array}$ & $\begin{array}{l}\text { Time, in } \\
\text { days, from } \\
\text { respiratory } \\
\text { symptoms } \\
\text { to ICU } \\
\text { admission }\end{array}$ & $\begin{array}{l}\text { Ventilatory } \\
\text { support }\end{array}$ & Superinfection & $\begin{array}{l}\text { Anti-infectious } \\
\text { agents }^{\mathrm{a}}\end{array}$ & Outcome \\
\hline 1 & $\begin{array}{l}\text { Chronic myeloid } \\
\text { leukemia }\end{array}$ & No & No & 4,000 & 3 & NIV & $\begin{array}{l}\text { Clinically } \\
\text { documented }\end{array}$ & C3G/macrolide & Alive \\
\hline 2 & $\begin{array}{l}\text { Allogeneic BMT } \\
\text { (12 months ago) } \\
\text { with GVHD }\end{array}$ & No & $\begin{array}{c}\text { Yes } \\
\text { (steroid/Cls) }\end{array}$ & 800 & 1 & MV & $\begin{array}{l}\text { Clinically } \\
\text { documented }\end{array}$ & $\begin{array}{l}\text { Piperacillin/ } \\
\text { FQ }\end{array}$ & Dead \\
\hline 3 & $\begin{array}{l}\text { Allogeneic BMT } \\
\text { (15 months ago) } \\
\text { with GVHD }\end{array}$ & No & $\begin{array}{c}\text { Yes } \\
\text { (steroid/Cls) }\end{array}$ & 600 & 1 & NIV & $\begin{array}{l}\text { Escherichia coli }+ \\
\text { Aspergillus fumigatus }\end{array}$ & $\begin{array}{l}\text { Imipeneme/ } \\
\text { FQ }\end{array}$ & Dead \\
\hline 4 & $\begin{array}{l}\text { Autologous BMT } \\
\text { for multiple myeloma }\end{array}$ & Yes & $\begin{array}{c}\text { Yes } \\
\text { (steroid) }\end{array}$ & 50 & 5 & None & $\begin{array}{l}\text { Clinically } \\
\text { documented }\end{array}$ & $\begin{array}{l}\text { Piperacillin/ } \\
\text { macrolide }\end{array}$ & Alive \\
\hline 5 & Renal transplantation & (ste & $\begin{array}{c}\text { Yes } \\
\text { teroid/Cls/MMF) }\end{array}$ & 1,200 & 5 & MV & $\begin{array}{l}\text { Pseudomonas } \\
\text { aeruginosa }\end{array}$ & $\begin{array}{l}\text { Piperacillin/ } \\
\text { macrolides }\end{array}$ & Dead \\
\hline 6 & HIV & No & No & 1,800 & 3 & None & $\begin{array}{l}\text { Streptococcus } \\
\text { pneumoniae }\end{array}$ & $\begin{array}{l}\mathrm{C} 3 \mathrm{G} / \\
\text { macrolide }\end{array}$ & Alive \\
\hline 7 & $\begin{array}{l}\text { Autologous BMT } \\
\text { for multiple myeloma }\end{array}$ & Yes & $\begin{array}{c}\text { Yes } \\
\text { (steroid) }\end{array}$ & 100 & 5 & MV & $\begin{array}{l}\text { Clinically } \\
\text { documented }\end{array}$ & $\begin{array}{l}\text { Piperacillin/ } \\
\text { macrolide }\end{array}$ & Alive \\
\hline 8 & Myelodysplasia & Yes & $\begin{array}{l}\text { Yes } \\
\text { (steroid) }\end{array}$ & 2,000 & 3 & MV & E. coli & $\begin{array}{l}\text { Piperacillin/ } \\
\text { macrolide }\end{array}$ & Dead \\
\hline 9 & Mantle cell lymphoma & a No & No & 100 & 2 & NIV and MV & S.pneumoniae & C3G/macrolide & Alive \\
\hline 10 & $\begin{array}{l}\text { Solid organ } \\
\text { transplantation }\end{array}$ & No & $\begin{array}{c}\text { Yes } \\
\text { (steroid) }\end{array}$ & 2,000 & 2 & None & S.pneumoniae & C3G/macrolide & Alive \\
\hline
\end{tabular}

${ }^{a}$ All patients were receiving oseltamivir. BMT, bone marrow transplantation; C3G, third-generation cephalosporin; $\mathrm{Cl}$, calcineurin inhibitor; FQ, fluoroquinolone; GVHD, graft-versus-host disease; ICU, intensive care unit; MMF, mycophenolate mofetil; MV, mechanical ventilation; NIV, non-invasive mechanical ventilation.

with influenza viruses [5] as the main defense mechanism is CD8 T-lymphocyte-mediated cytotoxicity. The clinical presentation in our patients was similar to that described in immunocompetent individuals, with symptom onset 4 days before ICU admission [2,4]. Mortality was high (40\%) compared with the overall population with H1N1 2009 infection [2-4]. The ICU stay was shorter than in the overall ICU population but the hospital stay was longer, perhaps because of prolonged viral shedding in lymphopenic patients [5].

\section{Abbreviations}

ICU, intensive care unit; IQR, interquartile range.

\section{Competing interests}

EA is a member of the French and European boards of Pfizer Inc (New York, NY, USA) and Gilead (Foster City, CA, USA), respectively. The other authors declare that they have no competing interests.

\section{Author details}

'Medical Intensive Care Unit, Saint-Louis University Hospital, APHP, 1 Avenue Claude Vellefaux, 75010 Paris, France. ${ }^{2}$ Paris-7 Paris-Diderot University, UFR de Médecine, 1 Avenue Claude Vellefaux, 75010 Paris, France. ${ }^{3}$ Virology Unit, Saint-Louis University Hospital, APHP, 1 Avenue Claude Vellefaux, 75010 Paris, France. ${ }^{4}$ Medical Intensive Care Unit, Hôtel Dieu University Hospital, APHP, 1 Avenue Claude Vellefaux, 75010 Paris, France.

Published: 14 April 2010

\section{References}

1. Fishman JA: Infection in solid-organ transplant recipients. N Engl J Med 2007, 357:2601-2614

2. Kumar A, Zarychanski R, Pinto R, Cook DJ, Marshall J, Lacroix J, StelfoxT, Bagshaw S, Choong K, Lamontagne F, Turgeon AF, Lapinsky S, Ahern SP, Smith $O$, Siddiqui F, Jouvet P, Khwaja K, Mclntyre L, Menon K, Hutchison J, Hornstein D, Joffe A, Lauzier F, Singh J, Karachi T, Wiebe K, Olafson K, Ramsey C, Sharma S, Dodek P, et al.: Critically ill patients with 2009 influenza A(H1N1) infection in Canada. JAMA 2009, 302:1872-1879.

3. Louie JK, Acosta M, Winter K, Jean C, Gavali S, Schechter R, Vugia D, Harriman K, Matyas B, Glaser CA, Samuel MC, Rosenberg J, Talarico J, Hatch D; California Pandemic (H1N1) Working Group: Factors associated with death or hospitalization due to pandemic 2009 influenza $A(\mathrm{H} 1 \mathrm{~N} 1)$ infection in California. JAMA 2009, 302:1896-1902.

4. ANZIC Influenza Investigators, Webb SA, Pettilä V, Seppelt I, Bellomo R, Bailey M, Cooper DJ, Cretikos M, Davies AR, Finfer S, Harrigan PW, Hart GK, Howe B, Iredell JR, McArthur C, Mitchell I, Morrison S, Nichol AD, Paterson DL, Peake S, Richards B, Stephens D, Turner A, Yung M: Critical care services and 2009 H1N1 influenza in Australia and New Zealand. N Engl J Med 2009, 361:1925-1934.

5. Vilchez RA, McCurry K, Dauber J, Lacono A, Griffith B, Fung J, Kusne S: Influenza virus infection in adult solid organ transplant recipients. Am J Transplant 2002, 2:287-291.

doi:10.1186/cc8927

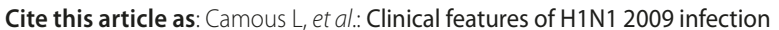
in critically ill immunocompromised patients. Critical Care 2010, 14:139. 